\title{
Clinical Image on Obstructed Labor in Mearg General Hospital West Tigray, Ethiopia
}

\section{Solomon Gebre*}

Mearg General Hospital, Emergency Surgical Department, Dansha, West Tigray, Ethiopia

${ }^{*}$ Corresponding author: Solomon Gebre, Mearg General Hospital, Emergency Surgical Department, Dansha, West Tigray, Ethiopia, Tel: 251911366834; Email:solomongebre300gmail.com

Received date: April 28, 2017; Accepted date: May 03, 2017; Published date: May 10, 2017

Copyright: (C) 2017 Gebre S, et al. This is an open-access article distributed under the terms of the Creative Commons Attribution License, which permits unrestricted use, distribution, and reproduction in any medium, provided the original author and source are credited.

Citation: Gebre S (2017) Clinical Image on Obstructed Labor in Mearg General Hospital West Tigray, Ethiopia. J Gen Pract (Los Angel) 5: i003. doi: 10.4172/2329-9126.1000i003

\section{Introduction}

This a 37 year old $\mathrm{G}=7 \mathrm{P}=6$ women with a $\mathrm{GA}=38 \mathrm{wk}+2$ presented with pushing down labor pain of three days duration after she referred from nearby health center, she had six alive babies who was born at home, she had no ANC follow-up, on physical examination her vital sign were $\mathrm{BP}=99 / 60 \mathrm{PR}=120 \mathrm{bpm} \mathrm{T}^{\circ}=39.3 \mathrm{RR}=32 \mathrm{bpm}$ and pertinent finding were dry bucal mucosa, fast and shallow breathing, Term size gravidi uterus with "three tumor abdomen", $\mathrm{FHB}=192 \mathrm{bpm}$ with titanic uterine contractions, edematous vulva on PV CX=Fully dilated station=+1 Grade 3 MSAF exasive caput and milding and difficult to insert urinary catheter Investigations $\mathrm{Bg}=\mathrm{O}-\mathrm{Hct}=40 \% \mathrm{U} / \mathrm{A}=\mathrm{NA}$ (no urine sample).

\section{Intraoperative Findings}

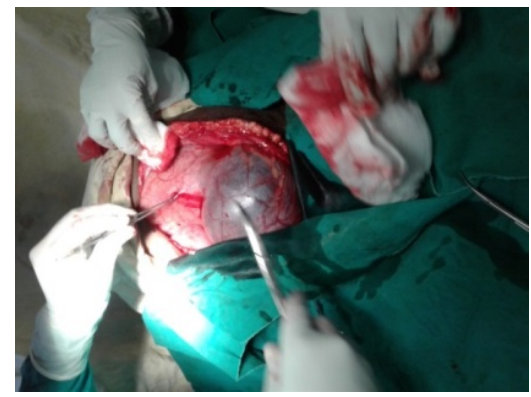

Figure 1: Distended bladder.

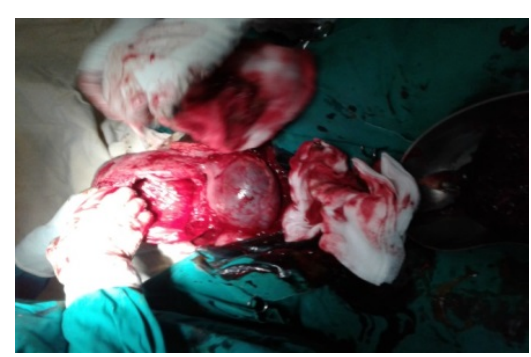

Figure 2: Vertical/Classical uterine incision.

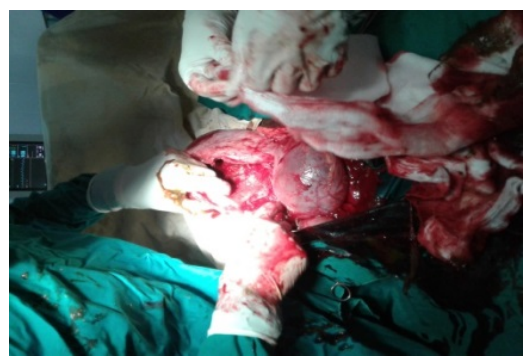

Figure 3: Mopping endometrial cavity.

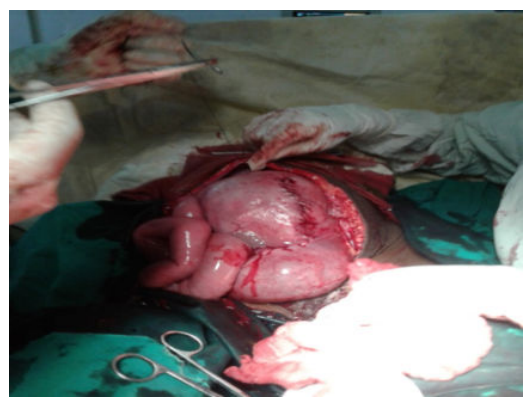

Figure 4: Repairing uterine incision.

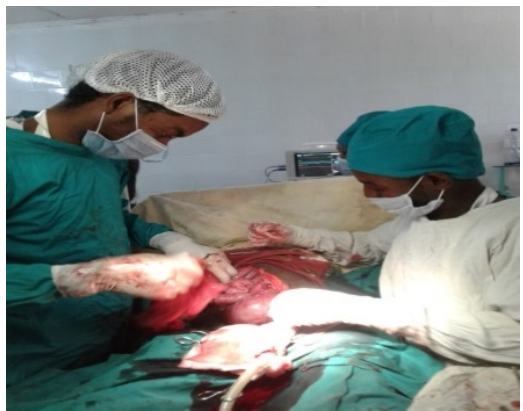

Figure 5: Closing the abdominal wall. 